\title{
The effect of Mo crystallinity on diffusion through the Si-on-Mo interface in EUV multilayer systems
}

\author{
S. Bruijn ${ }^{1}$, R.W.E. van de Kruijs ${ }^{1}$, A.E. Yakshin ${ }^{1}$, F. Bijkerk ${ }^{1,2}$ \\ ${ }^{1}$ FOM Institute for Plasma Physics Rijnhuizen, Edisonbaan 14, 3439 MN, Nieuwegein, The Netherlands, \\ www.rijnhuizen.nl \\ ${ }^{2}$ MESA + Institute for Nanotechnology, University Twente, Enschede, The Netherlands \\ s.bruijn@rijnhuizen.nl
}

Keywords: EUVL, multilayers, interfaces, amorphous Mo, polycrystalline $\mathrm{Mo}, \mathrm{Si}_{3} \mathrm{~N}_{4}$

\begin{abstract}
Thermally induced diffusion through the Si-on-Mo interface of multilayers with either amorphous or polycrystalline Mo layers has been investigated using grazing incidence and wide angle x-ray reflectometry. Diffusion through the Mo-on-Si interface was reduced by applying a diffusion barrier, allowing us to probe the diffusion at the opposite, Si-on-Mo interface. We found that diffusion through this interface is much slower for polycrystalline Mo than for amorphous Mo layers. The reason for this difference might be the larger defect concentration in amorphous Mo as compared to crystalline Mo.
\end{abstract}

\section{Introduction}

Extreme ultraviolet lithography (EUVL), designed to operate at a wavelength of around $13.5 \mathrm{~nm}$, is a serious candidate for next generation projection lithography systems to be applied in the semiconductor industry [1]. The optics in EUVL systems contain reflective multilayer coatings basically consisting of alternating Mo and Si layers. The theoretical multilayer reflection for $13.5 \mathrm{~nm}$ is $74 \%$ : in practice the reflectivity is limited to below $70 \%$ due to interface roughness as well as interaction of $\mathrm{Mo}$ and $\mathrm{Si}$ at the interfaces, where a molybdenum silicide is formed [2]. This molybdenum silicide formation is known to further accelerate at the enhanced temperatures that multilayers are exposed to under high intensity EUV illumination, leading to further reduction of the reflectivity as well as a change in the period of the multilayer. This causes the wavelength where maximum reflectivity occurs to deviate from the target value of $13.5 \mathrm{~nm}$.

Improvement of $\mathrm{Mo} / \mathrm{Si}$ coatings in this respect requires fundamental knowledge on the structure of the deposited layers as well as a detailed understanding of the processes that take place at the interfaces during deposition. It is already known from literature that amorphous Si layers are formed during deposition, whereas the Mo layer crystallizes above a critical thickness of about $2 \mathrm{~nm}[3,4]$. For crystalline Mo, it is also known that the formed Mo-on-Si interface is about $1 \mathrm{~nm}$ thick, while the formed Si-on-Mo interface is about $0.5 \mathrm{~nm}$ thick. When Mo is amorphous however, both interfaces are about $1 \mathrm{~nm}$ thick $[5,6,7]$.

Knowledge on the exact nature of interface formation is critical in understanding and controlling the processes that occur at the enhanced temperatures to which multilayer coatings will be exposed during EUV illumination. Previous studies on the thermal behaviour of Mo/Si multilayers showed that when Mo is crystalline the growth of the interface due to diffusion at the interfaces is much faster through the thick Mo-on-Si interface than through the thin Si-on-Mo interface [5]. As high temperature applications of multilayers might require thick diffusion barriers, the Mo layer thickness generally needs to be reduced to maximize the reflectivity and this may bring the Mo layer thickness 
below the critical thickness value for crystallization. In this paper we report on a study of the diffusion speed through the Si-on-Mo interface in multilayers with amorphous Mo layers. We do this by applying a $\mathrm{Si}_{3} \mathrm{~N}_{4}$ barrier which has shown to reduce diffusion at the Mo-on-Si interface [4] and is used as an example barrier in this study. The single sided applied barrier allows us to uniquely isolate the diffusion phenomena to one type of interface only. This is required in view of the asymmetry described above between the two types of interfaces.

\section{Experimental}

All samples were prepared by electron-beam evaporation, using a coater with a controlled UHV environment (base pressure $<2 \cdot 10^{-8}$ mbar). The thicknesses of the layers are controlled by an in-situ $\mathrm{X}$-ray reflectometer that monitors $\mathrm{X}$-ray reflection of the sample during multilayer growth. In addition, quartz mass balances are used to monitor layer thicknesses during the deposition. The sample holder rotates at $1 \mathrm{~Hz}$ to ensure a homogeneous coating in the azimuth direction. A detailed description of the setup can be found in [8]. The multilayers were deposited on $25 \times 25 \mathrm{~mm}^{2}$ super-polished silicon substrates.

Multilayers with different Mo thicknesses are made in order to obtain both crystalline and amorphous Mo layers. A deposition flux shaping mask was used during the deposition of Mo in order to create a linear Mo thickness gradient from 3.6nm-1.6nm on the samples mounted on the holder. To reduce the interface roughness during deposition, every Si layer was smoothened using a $\mathrm{Kr}$ ion beam. After polishing, the $\mathrm{Si}$ thickness $4.5 \mathrm{~nm}$. The $\mathrm{Si}_{3} \mathrm{~N}_{4}$ interface on $\mathrm{Si}$ was created by ion treatment with nitrogen directly after the $\mathrm{Kr}$ surface smoothening. More details on the process of nitride formation and the actually formed stoichiometries will be presented elsewhere.

To characterize the diffusion speeds through Mo-on-Si interface, all samples were sequentially annealed in a vacuum environment $\left(<10^{-5} \mathrm{mbar}\right)$ for $48 \mathrm{~h}$ at temperatures between $300^{\circ} \mathrm{C}$ and $375^{\circ} \mathrm{C}$ with steps of $25^{\circ} \mathrm{C}$. This method is applied to accelerate the diffusion phenomena, since the diffusion scales exponentially with temperature [9]. It is already known [4] that annealing either sequentially or directly to a final temperature does not result in a noticeable difference in multilayer structure after annealing. After each $25^{\circ} \mathrm{C}$ step, the multilayer structure is analyzed using grazing incidence X-ray reflectivity (GIXR) on a Philips X'pert $\mathrm{Cu}-\mathrm{K}_{\alpha}$ diffractometer using a wavelength of $0.15406 \mathrm{~nm}$. The crystalline structure of the layers is investigated using wide angle X-ray diffraction (WAXRD) on the $X^{\prime}$ pert diffractometer. During WAXRD measurements an incident angle of $1^{\circ}$ is used to maximize diffracted intensity. The sample is rotated $20^{\circ}$ in the sample plane in order to suppress diffraction from the crystalline Si substrate.

\section{Results and discussion}

Figure 1 shows WAXRD spectra of $\mathrm{Mo} / \mathrm{Si}$ multilayers in which a $\mathrm{Si}_{3} \mathrm{~N}_{4}$ diffusion barrier is applied on the Mo-on-Si interface to reduce diffusion through this interface. For Mo thicknesses of 2.2, 2.8 and $3.4 \mathrm{~nm}$, the spectra clearly indicate the formation of bcc polycrystalline Mo layers. For a Mo thickness of $1.4 \mathrm{~nm}$, the diffraction spectrum does not suggest polycrystalline Mo growth. We pose here that in this case predominantly amorphous Mo layers are formed.

Figure 2 shows the change in multilayer period as a function of the annealing temperature for multilayers with a single-sided $\mathrm{Si}_{3} \mathrm{~N}_{4}$ diffusion barrier and a multilayer without barriers as a reference. The diffusion through the Mo-on-Si interface is stopped, or at least hampered by the barrier. Therefore the change in multilayer period that is caused by diffusion is expected to be much smaller than that 


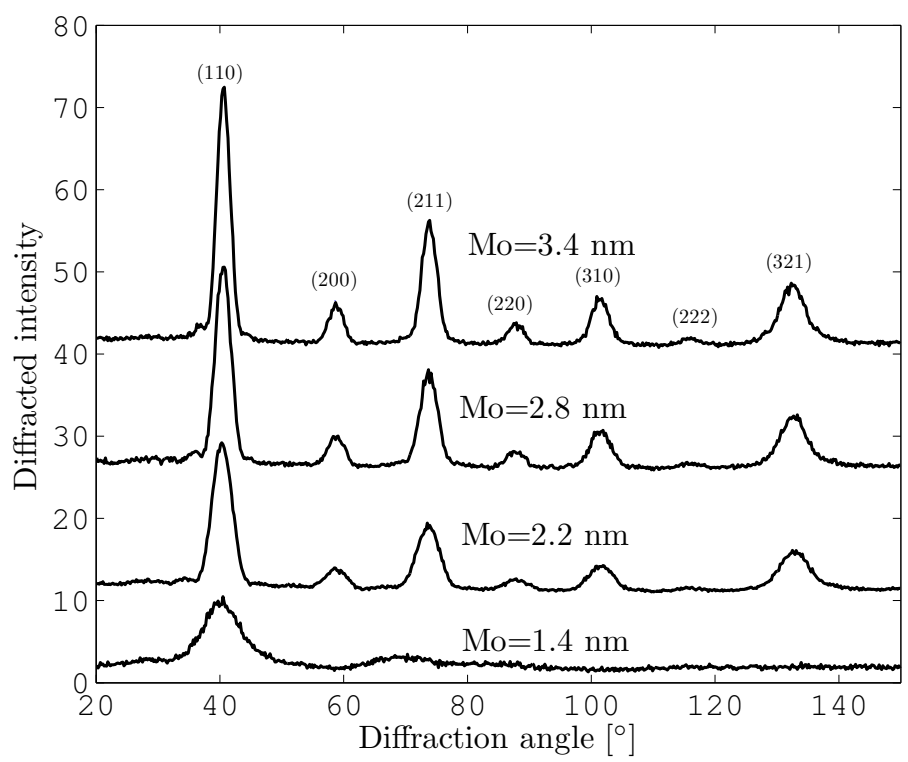

Figure 1: WAXRD spectra of as-deposited Mo/Si multilayers with $\mathrm{Si}_{3} \mathrm{~N}_{4}$ diffusion barriers at the Mo-on-Si interfaces. The spectra for $\mathrm{Mo}=2.2,2.8$ and $3.4 \mathrm{~nm}$ have been shifted for clarity. The top three spectra have been assigned to be crystalline Mo, the Miller indexes of the diffraction peaks are indicated above the spectrum the upper spectrum. The spectrum for $\mathrm{Mo}=1.4 \mathrm{~nm}$ does not correspond to bcc Mo.

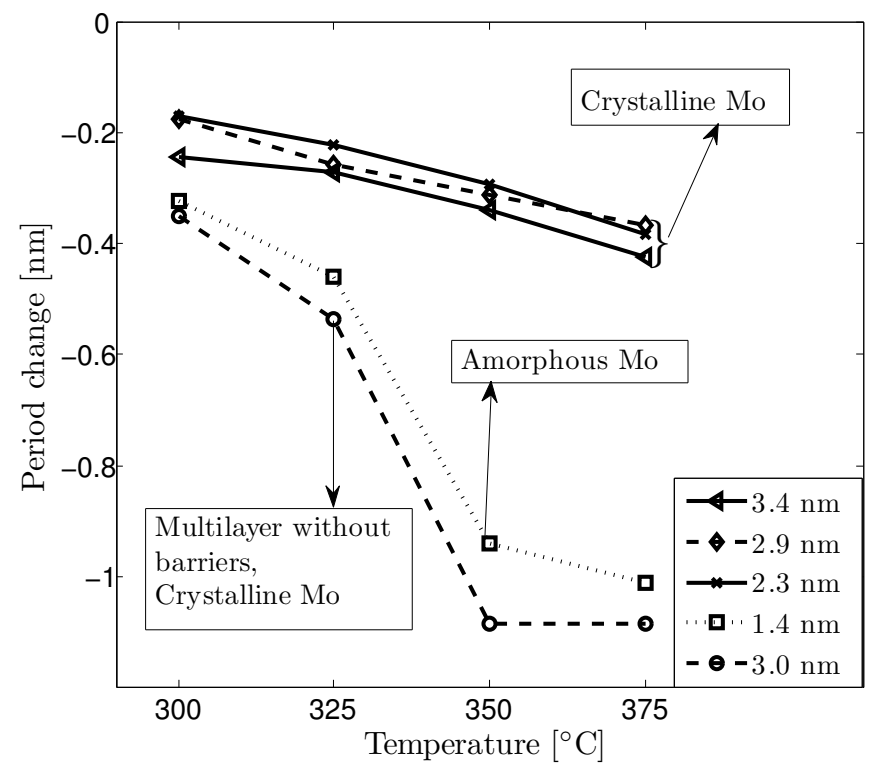

Figure 2: Period change as a function of annealing temperature for multilayers with $\mathrm{Si}_{3} \mathrm{~N}_{4}$ diffusion barriers at the Mo-on-Si interfaces. The samples in which the Mo is crystalline show less change than those in which Mo is amorphous, suggesting slower diffusion through the interfaces. The period change measured for a multilayer without any diffusion barrier is included as a reference. 
for a multilayer without barriers, because this interface has shown to grow slower than the Si-onMo in HRTEM studies [5]. For crystalline Mo, the period change is indeed reduced compared to a multilayer without diffusion barriers, as reported in [4]. In the case of amorphous Mo however, diffusion is equally fast for a multilayer without barriers. Clearly, diffusion through the Si-on-Mo interface is much faster when the Mo layer is amorphous.

This shows that indeed the crystallinity of the Mo layer has a large influence on the diffusion behaviour through the interfaces of a Mo/Si multilayer. The Si-on-Mo interface is thicker, and is a less effective diffusion barrier during thermal annealing when Mo is amorphous, than when Mo is crystalline.

A possible explanation for the observed differences in diffusion speed is the defect concentration in the Mo layer. It is a well known phenomenon that the diffusion speed through crystalline material is dependant on the amount of defects [10], since atoms are known to hop from one defect to the other. This makes diffusion through a polycrystalline material faster compared to diffusion through a fully crystalline lattice, because it has more defects around the grain boundaries. The amorphous state of materials has even more defects than a polycrystalline state and therefore it would be expected that in the amorphous state diffusion is faster.

\section{Conclusions}

We investigated the diffusion through the Si-on-Mo interface in multilayers with amorphous and polycrystalline Mo, depending on the initial thickness of the Mo layer in the multilayer samples. To study this phenomenon at a single type of interface, possible diffusion through the opposite, in this case the Mo-on-Si interface was reduced by applying a $\mathrm{Si}_{3} \mathrm{~N}_{4}$ diffusion barrier. The diffusion in the multilayers was investigated by thermal annealing followed by analysis using GIXR. We show a clear difference in diffusion speed through the Si-on-Mo interface for crystalline and amorphous Mo layers. When Mo is crystalline the diffusion through the Si-on-Mo interface is slow, but when the Mo is amorphous, the diffusion is considerably faster. The explanation posed for this difference is the higher defect concentration in amorphous Mo as compared to crystalline Mo.

\section{Acknowledgements}

This work is part of the FOM Industrial Partnership Programme I10 ('XMO') which is carried out under contract with Carl Zeiss SMT AG, Oberkochen and the 'Stichting voor Fundamenteel Onderzoek der Materie (FOM)' which is a part of the 'Nederlandse Organisatie voor Wetenschappelijk Onderzoek (NWO)'. We acknowledge the financial support from SenterNovem through the ACHieVE project.

\section{References}

[1] B. Wua and A. Kumar: J. Vac. Sci. Technol. B Vol. 25 (2007), p. 1743

[2] E. Louis, A.E. Yakshin, P.C. Goerts, S. Oestreich, R. Stuik, E.L.G. Maas, M.J.H. Kessels, F. Bijkerk, M. Haidl, S. Muellender, M. Mertin, S. Detlef, F. Scholze and G. Ulm: Proc. SPIE Vol. 3990 (2000), p. $406-411$

[3] R.W.E. van de Kruijs, E. Zoethout, A.E. Yakshin, and F. Bijkerk: Thin Solid Films Vol. 515 (2006), p. 430

[4] I. Nedelcu, R.W.E. van de Kruijs, A.E. Yakshin and F. Bijkerk: J. Appl. Phys. Vol.103 (2008), p. 083549 
[5] R.S. Rosen, D.G. Strearns, M.A. Viliardos, M.E. Kassner, S.P. Vernon ans Y.D. Cheng: Appl. Opt. Vol. 32 (1993), p. 6975

[6] S. Yulin, T. Feigl, T. Kuhlmann, N. Kaiser, A.I. Fedorenko, V.V. Kondratenko, O.V. Poltseva, V.A. Sevryukova, A.Y. Zolotaryov and E.N. Zubarev: J. Appl. Phys. Vol. 92 (2002), p. 2016

[7] S. Bajt, D.G. Stearns and P.A. Kearney: J. Appl. Phys. Vol. 90 (2001), p. 1017

[8] R. Schlatmann, C. Lu, J. Verhoeven, E.J. Puik and M.J. van der Wiel: Appl. Surf. Sci. Vol. 78 (1994), p. 147

[9] Martin Eden Glicksman: Diffusion in solids Field Theory, Solid-State Principles and Application (John Wiley \& Sons, inc., New York 2000)

[10] J.M. Poate, K.N. Tu, J.W. Mayer: Thin Films-Interdiffusion and Reactions (John Wiley \& Sons, inc., New York 1978) 